\title{
Trials to Improve Image Quality in Electron Tomography
}

T. Hashimoto, T. Kubo, T. Yotsuji, T. Oyagi, M. Kawasaki, E. Nakazawa, A. Wakui, T. Tanaka and H. Kobayashi

Naka Division, Hitachi High-Technologies Co. Ibaraki 312-8504 Japan

Electron tomography using Transmission Electron Microscope (TEM) / Scanning Transmission Electron Microscope (STEM) is widely used in medical science, biology, development of polymer materials, semiconductor failure analysis, etc. Electron tomography function is commonly composed of three processes: acquisition of a set of sequentially tilted images, three dimensional (3D-) reconstruction process and 3D-data displaying. As taking about 100 images with constant increment of specimen tilt and constant amount of defocus is rather harder for skilled operator, image acquisition should be automated. This process is performed by feedback system of acquired image to instrument control with TEM/STEM software.

For handling sets of sequentially tilted images, 3D-reconstruction calculation, and 3D-data displaying processes, Hitachi released image archiving and processing software EMIP-3D [1]. For 3D-reconstruction, the Filtered Back Projection (FBP) algorithm is contained as a standard component. Other calculation methods; Simultaneous Iterative Reconstruction Technique (SIRT) [2] [3], Topography Based Reconstruction Technique (TBRT) [1] [4], and Double-Axis Tomography (DAT) are optional. Calculated 3D-data can be displayed in arbitrary section or volume-rendering image in viewer function of the EMIP-3D.

Due to limitation of specimen tilt range, wedge shaped region of information is lost in image acquisition. The FBP or SIRT are generally used for 3D-reconstruction, but missing wedge artifact is inevitable for reconstructed results calculated with these methods. To reduce the artifact in electron tomography, there are several solutions; A) Expanding specimen tilting range to $+/-90$ degree, B) Adding restriction condition of specimen distribution or structure to improve calculation precision, and C) Making up lost information with data acquired in different way. For Solution A, we developed 3D analytical specimen holder that enables to acquire images of Focused Ion Beam processed micro-pillar shaped specimen. The HD-2700 STEM is able to add auto image acquisition function using this holder. The TBRT corresponds to the solution B. The TBRT algorithm uses topographic analysis by stereo-photogrammetry. 3 images with tilted angel of $\theta-5$, $\theta$, and $\theta+5$ degrees are picked up. X-Y coordinates of selected points are fixed in the image of $\theta$ degrees. Using images of $\theta-5$ and $\theta+5$ degrees, $Z$ coordinates of selected points are calculated. By restricting distribution in the SIRT algorithm based on calculated $Z$ coordinates of selected points, reconstruction with smaller artifact is calculated. Figure 1 shows comparison of TBRT and SIRT results of a replica specimen. The DAT is a solution C. For 120kV TEM H-7650 and HT7700, DAT solutions were developed by combination of specimen rotation holder and DAT option of the EMIP 3D. The specimen rotation holder enables to set specimen on one tilt axis and on another axis which is perpendicular to the first. Two sets of sequentially tilted images around the first axis and the second axis are acquired. Each set of images is reconstructed separately. Two 3D reconstructed discrete data are aligned their position and direction for summing up. As Characteristic points of the specimen are selected from image profile, application of fiducial marker is not necessary for DAT of the EMIP-3D. Figure 2 shows 3D-reconstruction of latex balls with FBP around tilt axis A and B and their summation with DAT function. Figure 3 is theoretical comparison of missing-wedge ratio [5]. 3D-reconstructed data with smaller artifact can be obtained with TBRT or DAT of the EMIP-3D. 


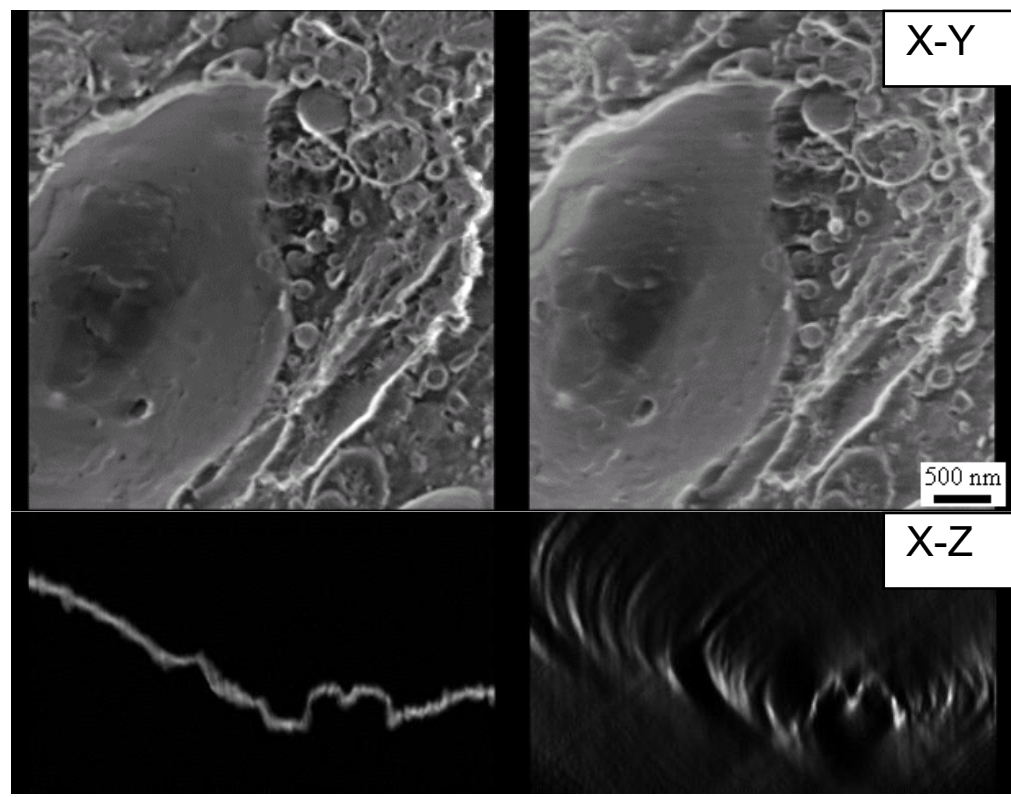

TBRT

SIRT
FIG. 1. Comparison of TBRT and SIRT results of a replica specimen. Artifact is much smaller in $\mathrm{X}-\mathrm{Z}$ section of TBRT than that of SIRT.

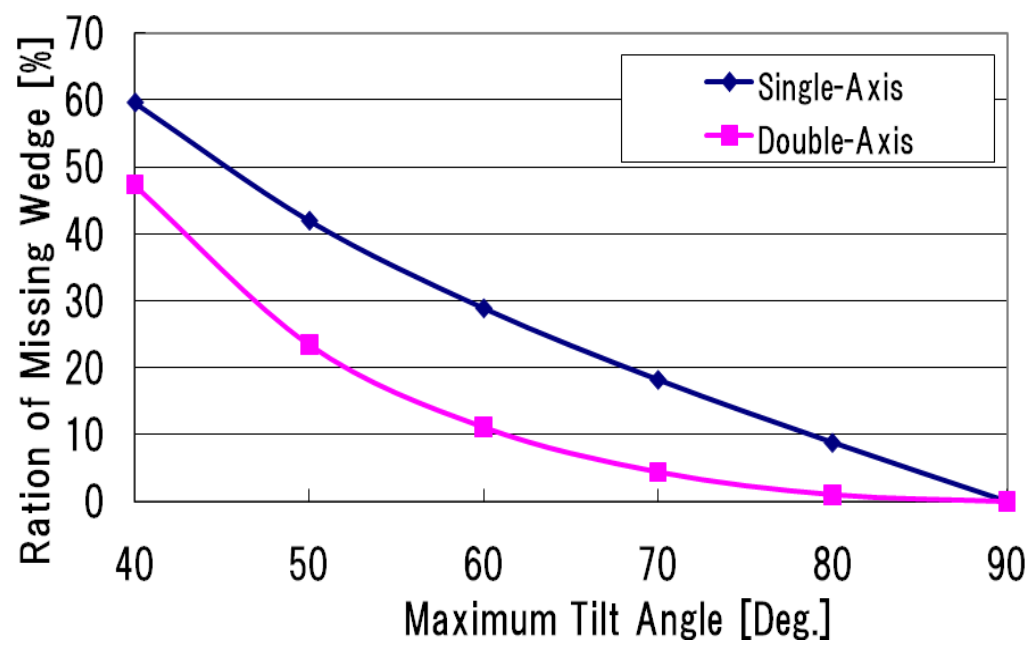

FIG. 3. Theoretical Comparison of Ratio of the

Missing Wedge Region in Single-Axis and DoubleAxis Tomography. [5]
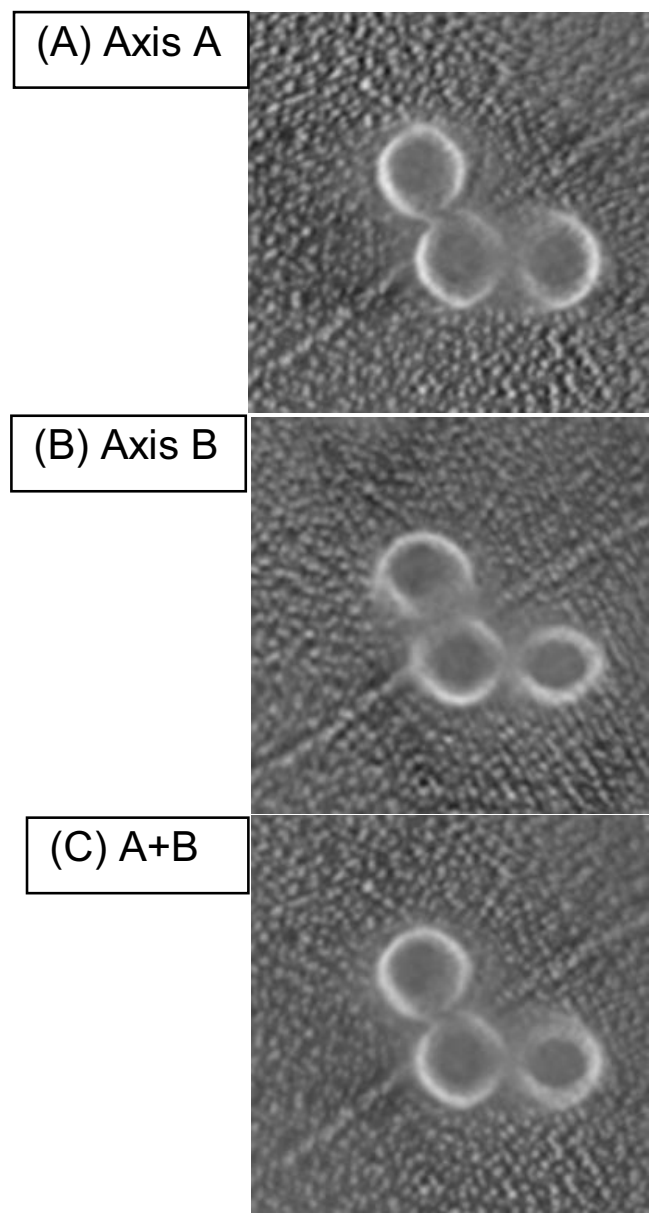

FIG. 2. FBP Reconstruction images of latex balls acquired around (A) the first "A" axis, and (B) another "B" axis perpendicular to the "A". (C) Summation of (A) and (B) with DAT function. (A) has up-down direction artifacts and (B) has left-right direction artifacts, but they are moderated in $(\mathrm{C})$.

\section{References}

[1] E. Nakazawa, et al, Proc. IMC16 Sapporo (2006) 696,

[2] M. Radermacher, in Harder, Donat-P.(Ed.), Image Analysis - Methods and Applications - ,CRC Press, Boca Raton, (2001) 295-327,

[3] E.T. O'Toole, et al., Mol. Biol. Cell, 10, (1999) 2017-2031,

[4] N. Baba, E. Katayama, Ultramicroscopy 108 (2008) 239-255,

[5] P.A. Penczek, J. Fraank, ELECTRON TOMOGRAPHY second edition, Springer,(2006), 319. 